\title{
Repeat Vaginal Natural Orifice Transluminal Endoscopic Surgery: A First Feasibility Study
}

\author{
Katrien Nulens Ilse Van Genechten Jan Baekelandt \\ Department of Gynecology and Obstetrics, Imelda Hospital, Bonheiden, Belgium
}

\section{Keywords \\ Repeat vaginal natural orifice transluminal endoscopic surgery · Colpotomy - Benign gynaecologic surgery . \\ Hysterectomy - Vaginally assisted natural orifice transluminal endoscopic surgery hysterectomy}

\begin{abstract}
Objectives: Vaginal natural orifice transluminal endoscopic surgery (vNOTES) is an emerging minimally invasive technique in benign gynaecologic surgery whereby surgical access to the peritoneal cavity is achieved through natural orifices, namely through a vaginal colpotomy. Experience in repeat vNOTES cases is limited and so far, repeat vNOTES cases have not been described in the literature. The purpose of this study was to demonstrate the feasibility of VNOTES hysterectomy in women with a history of previous VNOTES adnexal surgery. Design: We performed a retrospective cohort study of the first 11 repeat VNOTES cases in our centre. All vNOTES procedures, primary adnexal surgery as well as repeat vNOTES hysterectomy, were performed by one surgeon (J.B.). Materials, Setting, Methods: Between March 2016 and May 2020, 11 patients underwent a vNOTES hysterectomy after prior vNOTES adnexectomy or cystectomy in Imelda Hospital, Bonheiden, Belgium. Relevant patient characteristics and outcome data were collected after written informed consent. Results: Median age was 49 years (range
\end{abstract}

44-65) at the moment of the first vNOTES procedure. Two patients had one or more caesarean sections in history, and 2 women were nulliparous. Median interval between primary and repeat VNOTES procedure was 15 months (range 0.837 months). All patients underwent a vaginally assisted NOTES hysterectomy (VANH) as repeat vNOTES procedure. Performing a colpotomy and entering the peritoneal cavity after prior vNOTES was technically feasible in all cases. All VANHs were successfully performed. There were 3 minor complications after repeat VNOTES, of which one was anaesthesia-related. The 2 complications associated with the surgical procedure were both cystitis. In one of these 2 patients, there were high post-void residues, which were easily managed by bladder training. There were no conversions to laparoscopy or laparotomy, neither serious nor life-threatening complications. No ureteric, bladder, or intestinal injuries have occurred. Limitations: The retrospective design and small sample size are the main limitations of this study. Moreover, the follow-up period of the most recently operated patients was too short to draw conclusions on longterm outcomes, including sexual function. Conclusions: In all patients in this case series, VNOTES hysterectomy after prior VNOTES adnexal surgery was successfully performed. Large-scale prospective trials with long-term follow-up are needed to evaluate the safety and feasibility of multiple consecutive vNOTES procedures in 1 patient.

다 2021 S. Karger AG, Basel

$\begin{aligned} & \text { karger@karger.com } \\ & \text { www.karger.com/goi }\end{aligned}$
Karger ${ }^{\prime /}$

Katrien Nulens

Department of Gynecology and Obstetrics Imelda Hospital

Imeldalaan 9, BE-2820 Bonheiden (Belgium)

katrien_nulens@hotmail.com 


\section{Introduction}

The surgical approach for benign gynaecologic indications has changed markedly in the last decades with an increase in minimally invasive techniques and a concurrent decrease in associated morbidity $[1,2]$. Laparoscopy has become a standard technique to perform a hysterectomy, with recent attempts to further minimalize invasiveness [3]. Natural orifice transluminal endoscopic surgery (NOTES) is one of the newest evolutions in minimally invasive surgery whereby natural orifices are used to gain access to the abdominal cavity without visible scars [4]. NOTES has been adopted by general surgeons, urologists, gynaecologists, and gastro-enterologists and has shown to be a feasible and safe technique [5]. In benign gynaecology, the vaginal NOTES (vNOTES) technique has gained popularity over the past few years. The first randomized controlled trial comparing vNOTES to laparoscopic hysterectomy showed non-inferiority in successfully performing a hysterectomy without conversion. Moreover, there was a shorter hospital stay with more patients leaving the hospital within $12 \mathrm{~h}$ after surgery and less postoperative pain after vNOTES compared to laparoscopy [6]. A vaginally assisted NOTES hysterectomy (VANH) starts as a conventional vaginal hysterectomy. After anterior and posterior colpotomy and transection of both uterosacral ligaments, hysterectomy (with or without adnexectomy) is continued endoscopically through a vNOTES port [7]. In vNOTES adnexal surgery (adnexectomy, cystectomy, or salpingectomy), a colpotomy in the posterior vaginal fornix is used to directly access the pelvis through the pouch of Douglas. A small vNOTES port is then placed through the posterior fornix to enter endoscopic instruments into the peritoneal cavity [8]. The technique of colpotomy has been studied in the context of transvaginal specimen removal and seems to be safe and feasible with a minimal complication risk $[9,10]$. Moreover, previous studies did not show a negative effect on sexual function in women, neither an increased risk for adhesions [9]. However, whether vNOTES is technically feasible after previous surgery using colpotomy has not been described. To our knowledge, repeat vNOTES cases have never been reported in literature. This series of repeat vNOTES cases is in line with the IDEAL recommendations on the development of new surgical techniques (stage 1, innovation).
Table 1. Patient characteristics

\begin{tabular}{llllll}
\hline Case no. & Age, years & BMI, $\mathrm{kg} / \mathrm{m}^{2}$ & Parity & $\mathrm{VD}, n$ & $\mathrm{CS}, n$ \\
\hline 1 & 47 & 17.5 & 1 & 1 & 0 \\
2 & 48 & 26.6 & 2 & 1 & 1 \\
3 & 45 & 20.2 & 2 & 2 & 0 \\
4 & 49 & 23.0 & 2 & 2 & 0 \\
5 & 65 & 23.0 & 2 & 2 & 0 \\
6 & 48 & 25.2 & 0 & 0 & 0 \\
7 & 54 & 29.8 & 2 & 0 & 2 \\
8 & 56 & 24.7 & 2 & 2 & 0 \\
9 & 52 & 31.6 & 2 & 2 & 0 \\
10 & 50 & 22.5 & 2 & 2 & 0 \\
11 & 44 & 23.4 & 0 & 0 & 0 \\
\hline
\end{tabular}

VD, vaginal delivery; CS, caesarean section.

\section{Materials and Methods}

\section{Patients}

Between March 2016 and May 2020, 11 vNOTES hysterectomies were performed in patients with a history of vNOTES adnexectomy or vNOTES cystectomy in Imelda Hospital in Bonheiden, Belgium. Data from all women undergoing a vNOTES procedure are collected after written informed consent. Retrospectively, we analysed the following data from the 11 repeat vNOTES cases: age, BMI, gravidity, parity, number of vaginal deliveries, type of adnexal surgery (surgery no. 1), indication for adnexal surgery, indication for hysterectomy (surgery no. 2), interval between primary and repeat surgery, total operating time surgery no. 1 and 2, complications, postoperative visual analogue scale (VAS) pain scores, size of the adnexal mass (surgery no. 1), uterine weight (surgery no. 2), hospital stay, and the presence of adhesions. As this is a retrospective study, ethics approval was not required.

\section{Surgical Technique}

The surgical techniques have also been described in detail in the HALON [7] and NOTABLE [8] study protocol. In both vNOTES adnexal surgery and vNOTES hysterectomy, the patient is placed in lithotomy position after induction of general anaesthesia (GA). By rectovaginal examination, obliteration of the pouch of Douglas is excluded both in primary and repeat vNOTES procedures. The abdomen, pelvic area, and vagina are disinfected and draped. The bladder is emptied using a Foley catheter. At the end of each vNOTES procedure, after closure of the colpotomy or vaginal cuff, a plug is inserted in the vagina for haemostatic compression. The Foley catheter and the vaginal plug are both removed $3 \mathrm{~h}$ after the end of surgery $[7,8]$.

Vaginal Natural Orifice Transluminal Endoscopic Surgery Adnexectomy

For a vNOTES adnexectomy, the procedure starts with a $2.5 \mathrm{~cm}$ posterior colpotomy using a cold knife whereby the pouch of Douglas is opened using cold scissors [8]. The vNOTES port (Gelpoint vPath, Applied Medical, Rancho Santa Margarita, CA, USA) is inserted into the pouch of Douglas. After $\mathrm{CO}_{2}$ insufflation until a max- 
Table 2. Characteristics of primary adnexal surgery

\begin{tabular}{|c|c|c|c|c|c|c|c|}
\hline $\begin{array}{l}\text { Case } \\
\text { no. }\end{array}$ & Type of surgery no. 1 & Indication & $\begin{array}{l}\text { Total } \\
\text { operating } \\
\text { time, min }\end{array}$ & $\begin{array}{l}\text { Largest } \\
\text { diameter of } \\
\text { adnexal mass, } \\
\text { mm }\end{array}$ & $\begin{array}{l}\text { Perioperative } \\
\text { complications }\end{array}$ & $\begin{array}{l}\text { VAS pain } \\
\text { score after } \\
6 / 24 \mathrm{~h}\end{array}$ & $\begin{array}{l}\text { Hospital } \\
\text { stay, } \\
\text { days }\end{array}$ \\
\hline 1 & USO R + hysteroscopy + D\&C & Ovarian cyst $\mathrm{R}$, haemato-metrium & 35 & 50 & - & $2 / 2$ & 2 \\
\hline 2 & USO R + LLETZ & Ovarian cyst R + abnormal cervical smear & 40 & 45 & - & $2 /-$ & 1 \\
\hline 3 & USO L + TOT & Ovarian cyst L stress incontinence & 45 & 50 & - & $2 / 2$ & 3 \\
\hline 4 & USO L + endometrial ablation & Ovarian cyst L, menorrhagia & 60 & 40 & PONV & $1 / 3$ & 2 \\
\hline 5 & USO R & Ovarian cyst $\mathrm{R}$ & 30 & 55 & - & $1 / 1$ & 2 \\
\hline 6 & USO R & Ovarian cyst $\mathrm{R}$ & 45 & 90 & $\begin{array}{l}\text { Anaemia } \\
(\mathrm{Hb} 8.7 \mathrm{~g} / \mathrm{dL})\end{array}$ & $2 / 2$ & 2 \\
\hline 7 & $\begin{array}{l}\text { USO L + LLETZ + hysteroscopy + } \\
\text { D\&C }\end{array}$ & Ovarian cyst L, abnormal cervical smear & 40 & 55 & - & $2 / 1$ & 2 \\
\hline 8 & $\mathrm{BSO}+$ hysteroscopy $+\mathrm{D} \& \mathrm{C}$ & Ovarian cyst $\mathrm{R}+$ endometrial polyp & 60 & $\begin{array}{l}\text { R: } 20 \\
\text { L: } 18\end{array}$ & - & $0 /-$ & 1 \\
\hline 9 & $\mathrm{BSO}$ & Persisting ovarian cyst L, postmenopausal & 50 & $\begin{array}{l}\text { R: } 25 \\
\text { L: } 40\end{array}$ & - & $2 / 0$ & 2 \\
\hline 10 & Cystectomy R & Ovarian cyst R & 60 & 35 & - & $2 /-$ & 2 \\
\hline 11 & USO L & Ovarian cyst L & 42 & 140 & - & $1 / 1$ & 2 \\
\hline
\end{tabular}

VAS, visual analogue scale; h, hours; min, minutes; USO, unilateral salpingo-oophorectomy; BSO, bilateral salpingo-oophorectomy; R, right; L, left; LLETZ, large loop excision of transformation zone; TOT, transobturator tape; $\mathrm{Hb}$, haemoglobin; PONV, postoperative nausea and vomiting; $\mathrm{D} \& \mathrm{C}$, dilation and curettage.

imal intraperitoneal pressure of $10 \mathrm{~mm} \mathrm{Hg}$, a $10 \mathrm{~mm}$ rigid $30^{\circ}$ endoscope is inserted into the peritoneal cavity. The operating table is tilted in Trendelenburg position, the small intestine is lifted out of the pelvis, and the ureters are identified. The proximal end of the Fallopian tube is coagulated and transected. The ovarian and infundibulopelvic ligament are coagulated and cut to perform a unilateral salpingo-oophorectomy. Depending on the indication, the procedure can be repeated on the contralateral side for a bilateral salpingooophorectomy. When a cystectomy is performed, the cyst is dissected from the ovarian cortex and the ovary stays in place. Haemostasis is confirmed and the peritoneal cavity is rinsed. Small benign-looking adnexae can be removed directly through the wound protector part of the vNOTES port. Large adnexae or adnexae with uncertain biologic behaviour are removed in an endobag after aspiration of cyst fluid without intraperitoneal spilling. By using 3 interrupted figureof-eight Vicryl 2/0, the colpotomy is sutured.

Vaginal Natural Orifice Transluminal Endoscopic Surgery

Hysterectomy

All patients in this series underwent a VANH. The surgical procedure in these repeat vNOTES cases was identical to classic primary VANH. Pozzi tenaculum forceps are placed on the anterior and posterior cervical lip to provide caudal traction. Coldknife circumcision of the cervix is performed after submucosal infiltration of an adrenalin-ropivacaine solution (for hydrodissection and local anaesthesia). The vaginal mucosa is sheared off by a gauze compress. Then, the vesico-uterine peritoneum and the pouch of Douglas are opened with cold scissors by anterior and posterior colpotomy, respectively. Subsequently, the uterosacral ligaments are cut, as done in conventional vaginal hysterectomy. The vNOTES port is inserted into the peritoneal cavity to establish a pneumoperitoneum. This device enables several trocars to be inserted through a single port. Again, a $10 \mathrm{~mm}$ rigid $30^{\circ}$ laparoscope is used. Anatomic structures of the female pelvis, including the ureters, can be identified. The hysterectomy is performed by dissecting caudally to cranially using endoscopic instruments with bipolar coagulation. The Fallopian tubes are removed in all women after counselling; the ovaries only when indicated. At the end of the hysterectomy, the surgeon removes the vNOTES port and the uterus through the vagina. When the uterus is too large to extract vaginally, transvaginal cold-knife morcellation is performed. The vaginal cuff is closed as in a conventional vaginal surgery.

\section{Anaesthesia and Pain Management Protocol}

Standard induction of GA was performed by using intravenous (IV) propofol $2.5 \mathrm{mg} / \mathrm{kg}$, sufentanil $0.15 \mu \mathrm{g} / \mathrm{kg}$, rocuronium $0.6 \mathrm{mg} / \mathrm{kg}$, and dexamethasone $0.1 \mathrm{mg} / \mathrm{kg}$ (max $5 \mathrm{mg}$ ). Desflurane was used for maintenance of GA at a minimal alveolar concentration of 1 . During surgery, alfentanil $5 \mu \mathrm{g} / \mathrm{kg}$ was administered when indicated. Thirty minutes before the end of the procedure, all patients received IV paracetamol $1 \mathrm{~g}$ and ketoro- 


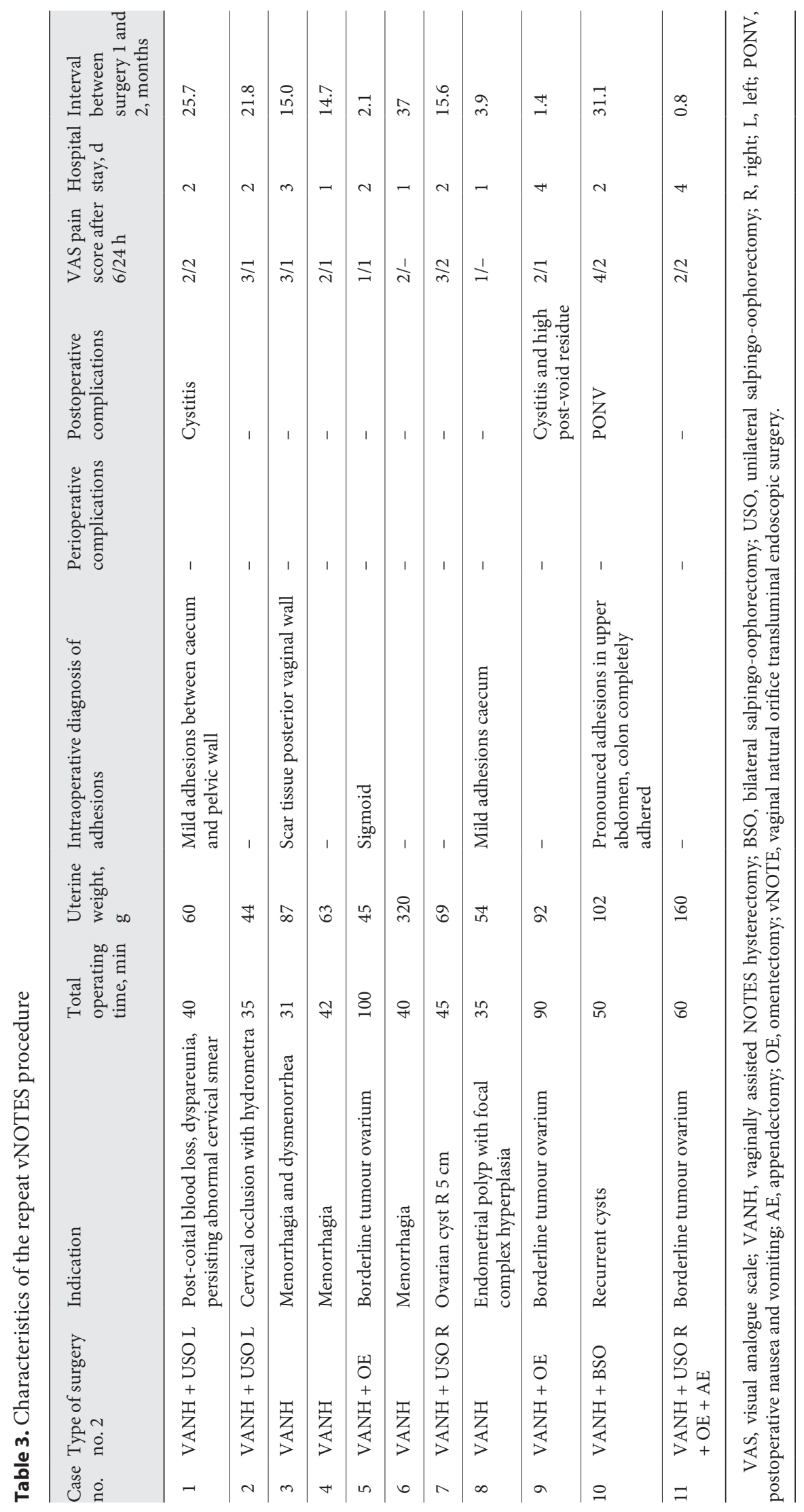


lac $0.5 \mathrm{mg} / \mathrm{kg}$ ( $\max 30 \mathrm{mg}$ ) with a maximum of $30 \mathrm{mg}$. When the patient reported a VAS pain score $>4$ on the recovery, $2.5 \mathrm{mg}$ piritramide IV was used. Postoperative pain is routinely assessed after 6 and $24 \mathrm{~h}$ by the nurse on the ward. Paracetamol $1 \mathrm{~g} \mathrm{IV}$ and ketorolac $30 \mathrm{mg}$ IV are the first-line analgesics for patients reporting a VAS score $>4$. Tramadol ( $300 \mathrm{mg}$ in $24 \mathrm{~h}$ ) is used in second line.

\section{Results}

Patient characteristics are shown in Table 1 . The median age was 49 years (range 44-65). The median BMI was $23.4 \mathrm{~kg} / \mathrm{m}^{2}$ (range 17.5-31.6). Two patients were nulliparous. One woman had had 2 caesarean sections and no vaginal deliveries. One patient had a normal vaginal delivery and a caesarean section. Characteristics of the primary adnexal surgery of the consecutive patients are summarized in Table 2.

Table 3 shows the surgical outcomes of the repeat vNOTES procedures (i.e., VANH) and the interval between primary and repeat vNOTES. The median interval between the 2 surgeries was 15 months (range 0.8-37 months). The mean operating time was 51 min. Mean uterine weight was $99 \mathrm{~g}$. Median VAS pain score $6 \mathrm{~h}$ after surgery was 2 (range 1-6) and 24 h after surgery 1.5 (range 1-2). Postoperatively, 2 patients had complications related to surgery. Cystitis was diagnosed in case number 1 and managed with antibiotics lege artis. In case 9 in $\mathrm{Ta}-$ ble 3 , there was a problem of incomplete bladder emptying with increased post-void residues due to cystitis, which resolved after bladder training and antibiotics. Moreover, one patient had postoperative nausea and vomiting, which was treated with IV anti-emetics.

\section{Discussion}

vNOTES is a relatively novel technique in the field of minimally invasive surgery that gained popularity in the past few years. In experienced hands, it is a safe and scarless alternative to vaginal, laparoscopic, and open surgery. Besides non-inferiority in successfully performing a hysterectomy, possible advantages of vNOTES hysterectomy over laparoscopy are shorter hospital stay and less postoperative pain [6]. Especially when nulliparity, obesity [11], or large uterine volume is present, all factors that may complicate the feasibility of a vaginal hysterectomy, vNOTES offers the advantages of endoscopic surgery with better visualization of pelvic anatomy. Moreover, the endoscopic access facilitates removal of the Fallopian tubes or ovaries during vNOTES compared to classic vaginal hysterectomy. In adnexal surgery via vNOTES (i.e., unilateral salpingo-oophorectomy, bilateral salpingooophorectomy, salpingectomy, or cystectomy), a colpotomy is made in the posterior vaginal fornix to enter the peritoneal cavity. This also means that vNOTES uses a vaginal incision into the pouch of Douglas that is not made via the conventional abdominal access (laparoscopy or laparotomy). Relative contraindications for vNOTES are severe pelvic adhesions (frozen pelvis), obliteration of the pouch of Douglas, and rectovaginal endometriosis $[12,13]$. Whether a previous colpotomy may complicate future surgery due to adhesions or scar tissue has not yet been studied in the light of vNOTES. However, there is plenty of experience in the use of a colpotomy for specimen extraction, and it has shown to be a safe and feasible technique with a very low complication rate $[9,10,14]$. Moreover, a colpotomy does not seem to increase the risk for later sexual dysfunction or postoperative adhesions although more research is needed.

In this case series, we describe the patient characteristics and surgical outcome of 11 women who underwent a vNOTES hysterectomy after prior vNOTES adnexectomy (in 10 cases) or cystectomy (1 case). To the best of our knowledge, repeat vNOTES cases have never been reported in the literature.

In this small case series, only 2 minor perioperative surgical complications where seen: 2 patients presented with postoperative cystitis. There were no conversions to laparoscopy or laparotomy. Exactly the same surgical steps were followed in these repeat vNOTES cases as in VANH cases without prior vNOTES surgery. During the repeat vNOTES procedure (VANH), the posterior colpotomy and the opening of the pouch of Douglas went smoothly in all cases. Intra-abdominal adhesions were present in varying degree, and mild fibrotic scar tissue at the colpotomy site was noticed in one case. However, there were no problems in accessing the peritoneal cavity due to the absence of relevant adhesions at the colpotomy site that interfered with safely performing a repeat colpotomy. vNOTES allowed easy adhesiolysis.

Postoperative VAS pain scores were low, both after primary and repeat vNOTES. This is in line with the findings of the first randomized controlled trial comparing VANH with laparoscopic hysterectomy, wherein vNOTES was associated with significantly lower pain scores [6]. The study design of a retrospective case series is an important limitation in the assessment of the safety and feasibility of repeat vNOTES. Moreover, all procedures were performed by one surgeon (J.B.) in a single 
centre, which is possibly less generalizable. Follow-up was limited due to the retrospective design, whereby long-term effects on sexual function, dyspareunia, pelvic, or vaginal pain cannot properly be assessed. Large, prospective studies are needed to further evaluate the immediate and long-term outcome of repeating vNOTES procedures in the same patient.

\section{Conclusion}

In this case series, vNOTES hysterectomy was safely and successfully completed in patients with a history of vNOTES adnexal surgery. Only 2 minor surgical complications (postoperative cystitis in patients) were reported. There were no conversions to laparoscopy or laparotomy. Neither were their significant adhesions after prior vNOTES that complicated the feasibility of entering the peritoneal cavity. This IDEAL stage 1 study is the first report on repeat vNOTES surgery and is only a first small step in the scientific evaluation process needed to validate repeat vNOTES. Large-scale prospective clinical trials are needed to further evaluate the safety and feasibility of repeat vNOTES procedures, as well as the long-term impact on sexual function in women.

\section{Statement of Ethics}

All patients have given their written informed consent for the anonymous processing of baseline characteristics and surgical outcome data for research purposes. Seen the retrospective design of this study, ethical approval was not required.

\section{Conflict of Interest Statement}

Dr. Jan Baekelandt discloses consultancy for Applied Medical. Dr. Katrien Nulens has no conflicts of interest to declare. Ilse Van Genechten has no conflicts of interest to declare.

\section{Funding Sources}

This study was conducted, and the manuscript was written without any financial support from a pharmaceutical company or any other third party.

\section{Author Contributions}

Dr. Jan Baekelandt was the gynaecologist performing all surgical procedures and was involved in the writing of the manuscript. Dr. Katrien Nulens was involved in the writing of the manuscript as first author. Ilse Van Genechten was involved in the writing of the manuscript.

\section{References}

1 Hansdottir K, Gudmundsson JA. Trends in minimally invasive hysterectomy techniques for nonmalignant conditions in a defined population. Gynecol Obstet Invest. 2018; 83(2):198-202

2 Chrysostomou A, Djokovic D, Edridge W, van Herendael BJ. Evidence-based guidelines for vaginal hysterectomy of the International Society for Gynecologic Endoscopy (ISGE). Eur J Obstet Gynecol Reprod Biol. 2018 Dec; 231:262-7.

3 Tchartchian G, Heldmann P, Bojahr B, Larbig A, De Wilde RL. The laparoscopic-assisted combined hysterectomy: a new surgical concept compared to the classical laparoscopicassisted vaginal hysterectomy by a prospective study. Gynecol Obstet Invest. 2017;82(3): 223-9.

4 Yang E, Nie D, Li Z. Comparison of major clinical outcomes between transvaginal NOTES and traditional laparoscopic surgery: a systematic review and meta-analysis. J Surg Res. 2019 Dec;244:278-90.

5 Rattner D, Kalloo A, ASGE/SAGES Working Group. ASGE/SAGES working group on natural orifice translumenal endoscopic surgery. October 2005. Surg Endosc. 2006 Feb;20(2): 329-33.
6 Baekelandt JF, De Mulder PA, Le Roy I, Mathieu C, Laenen A, Enzlin P, et al. Hysterectomy by transvaginal natural orifice transluminal endoscopic surgery versus laparoscopy as a day-care procedure: a randomised controlled trial. BJOG Int J Obstet Gynaecol. 2019 Jan;126(1):105-13.

7 Baekelandt J, De Mulder PA, Le Roy I, Mathieu C, Laenen A, Enzlin P, et al. HALONhysterectomy by transabdominal laparoscopy or natural orifice transluminal endoscopic surgery: a randomised controlled trial (study protocol). BMJ Open. 2016 Aug;6(8):e011546.

8 Baekelandt JF, De Mulder PA, Le Roy I, Mathieu C, Laenen A, Enzlin P, et al. Transvaginal natural orifice transluminal endoscopic surgery (vNOTES) adnexectomy for benign pathology compared with laparoscopic excision (NOTABLE): a protocol for a randomised controlled trial. BMJ Open. 2018 Jan;8(1):e018059.

9 Uccella S, Cromi A, Bogani G, Casarin J, Serati M, Ghezzi F. Transvaginal specimen extraction at laparoscopy without concomitant hysterectomy: our experience and systematic review of the literature. J Minim Invasive Gynecol. 2013 Sep-Oct;20(5):583-90.
10 Tolcher MC, Kalogera E, Hopkins MR, Weaver AL, Bingener J, Dowdy SC. Safety of culdotomy as a surgical approach: implications for natural orifice transluminal endoscopic surgery. JSLS. 2012 Jul-Sep;16(3):41320.

11 Panait L, Wood SG, Bell RL, Duffy AJ, Roberts KE. Transvaginal natural orifice transluminal endoscopic surgery in the morbidly obese. Surg Endosc. 2013 Jul;27(7):2625-9.

12 Baekelandt J, De Mulder PA, Le Roy I, Mathieu C, Laenen A, Enzlin P, et al. Postoperative outcomes and quality of life following hysterectomy by natural orifice transluminal endoscopic surgery (NOTES) compared to laparoscopy in women with a non-prolapsed uterus and benign gynaecological disease: a systematic review and meta-analysis. Eur J Obstet Gynecol Reprod Biol. 2017 Jan;208:6-15.

13 Lee CL, Wu KY, Su H, Ueng SH, Yen CF. Transvaginal natural-orifice transluminal endoscopic surgery (NOTES) in adnexal procedures. J Minim Invasive Gynecol. 2012 JulAug;19(4):509-13.

14 Clark LE, Menderes G, Tower AM, Silasi DA, Azodi M. A simple approach to specimen retrieval via posterior colpotomy incision. JSLS. 2015;19(2):e2015.00222. 